\title{
First characterization of a superconducting filter-bank spectrometer for hyper-spectral microwave atmospheric sounding with transition edge sensor readout
}

Cite as: J. Appl. Phys. 127, 244501 (2020); https://doi.org/10.1063/5.0002984

Submitted: 29 January 2020. Accepted: 03 June 2020 . Published Online: 22 June 2020

D. J. Goldie (D), C. N. Thomas (D), S. Withington (D), A. Orlando (D), R. Sudiwala (D), P. Hargrave (D), and P. K. Dongre (i)
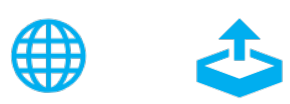

\section{ARTICLES YOU MAY BE INTERESTED IN}

Molecular and thermodynamics descriptions of flow-induced crystallization in semi-crystalline polymers

Journal of Applied Physics 127, 241101 (2020); https://doi.org/10.1063/5.0012376

\section{Lock-in Amplifiers up to $600 \mathrm{MHz}$}
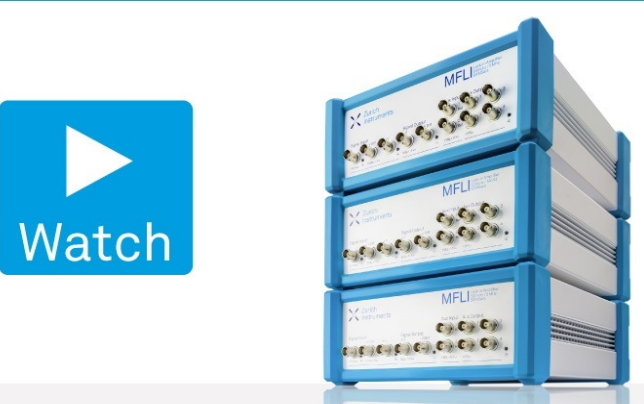


\title{
First characterization of a superconducting filter- bank spectrometer for hyper-spectral microwave atmospheric sounding with transition edge sensor readout
}

Cite as: J. Appl. Phys. 127, 244501 (2020); doi: 10.1063/5.0002984

Submitted: 29 January 2020 . Accepted: 3 June 2020 .

Published Online: 22 June 2020

D. J. Goldie, ${ }^{1, a)}$ (D) C. N. Thomas, ${ }^{1}$ (D) S. Withington, ${ }^{1}$ (D) A. Orlando, ${ }^{2}$ (D R. Sudiwala, ${ }^{2}$ (D) P. Hargrave, ${ }^{2}$ (D) and P. K. Dongre 2 (D)

\author{
AFFILIATIONS \\ ${ }^{1}$ Quantum Sensors Group, Cavendish Laboratory, University of Cambridge, JJ Thomson Avenue, Cambridge CB3 OHE, \\ United Kingdom \\ ${ }^{2}$ Astronomy Instrumentation Group, School of Physics and Astronomy, Cardiff University, Cardiff CF24 3AA, United Kingdom
}

${ }^{a)}$ Author to whom correspondence should be addressed: goldie@mrao.cam.ac.uk

\begin{abstract}
We describe the design, fabrication, integration, and characterization of a prototype superconducting filter bank with transition edge sensor readout designed to explore millimeter-wave detection at frequencies in the range of 40-65 GHz. Results indicate highly uniform filter channel placement in frequency and high overall detection efficiency. The route to a full atmospheric sounding instrument in this frequency range is discussed.
\end{abstract}

Published under license by AIP Publishing. https://doi.org/10.1063/5.0002984

\section{INTRODUCTION}

Superconducting on-chip filter-bank spectrometers (SFBSs) are a promising technology for a number of scientifically important applications in astronomy and meteorology that require low-noise, spectroscopic measurements at millimeter and sub-millimeter wavelengths. SFBSs are capable of achieving high channel counts and the individual channel characteristics such as shape, width, and position in frequency, power handling, and sensitivity can be tuned to the application. Moreover, the micro-fabrication techniques used in the production of these thin-film devices mean that SFBSs are intrinsically low-mass, physically compact, and easily reproducible, making them well-suited for array applications for both ground-based and satellite-borne instruments. High signal detection efficiencies can be achieved up to the superconducting pair-breaking threshold of the superconductors used in the design, typically $680 \mathrm{GHz}$ for $\mathrm{Nb}$ but higher for superconducting compounds such as $\mathrm{NbN}$ or NbTiN.

Applications in astronomy include surveys of moderate redshift galaxies $(Z=4-6)$ by precision determination of the frequencies of $\mathrm{CO}$ and $[\mathrm{CII}]$ rotational lines, multichroic pixels for cosmic microwave background (CMB) observations (foreground subtraction by observing in the $24-30$ and $30-48 \mathrm{GHz}$ atmospheric windows ${ }^{1}$ and simultaneous observation in multiple CMB frequency bands ${ }^{2,3}$ ), or observation of low-Z CO and O line emissions from nearby galaxies. ${ }^{4}$ Chip spectrometers coupling to superconducting kinetic inductance detectors (KIDs) are being developed by a number of groups and large multiplexing counts of order 1000s have been demonstrated. ${ }^{5,6}$ However, KIDs are difficult to design for detection at low frequencies $v \leqq 100 \mathrm{GHz}$, the pair-breaking threshold for a typical low superconducting transition temperature material such as $\mathrm{Al}^{7}$ and can also be challenging to calibrate as regard their power-tooutput-signal responsivity. Superconducting transition edge sensors (TESs) are a type of bolometric detector where the power absorption is not limited by the pair-breaking threshold of a superconducting film. In addition, their (power-to-current) responsivity is straightforward to determine both theoretically and experimentally. TES multiplexing schemes are a mature technology using both time- and frequency-domain approaches giving multiplexing factors of order $100 \mathrm{~s},{ }^{8,9}$ and microwave TES readout schemes promise to equal the multiplexing factors demonstrated for KIDs. ${ }^{10,11}$ 
Vertical profiles of atmospheric temperature and humidity measured by satellite-borne radiometric sounders provide vital information for long-range weather forecasting. These sounders work by measuring the upwelling radiance from the atmosphere in a number of spectral channels, typically either at microwave (MW) or infrared (IR) wavelengths. Vertical resolution and measurement accuracy improve rapidly with greater channel number and radiometric sensitivity. ${ }^{12,13}$ Significant progress has been made in IR sounder performance; the Infrared Atmospheric Sounding Interferometer (IASI), for example, provides over 8000 channels with sub-kelvin noise equivalent differential temperature (NETD) ${ }^{14}$ However, while able to provide high quality data, IR sounders can do so only under infrequent clear-sky conditions, as clouds absorb and interfere with the signal of interest. MW sounders, by contrast, are not affected by cloud cover, but their use has been hampered by poorer instrument performance. Channel number is a significant problem: the Advanced Microwave Sounding Unit-A (AMSU-A) in current use has, for example, only 15 channels, ${ }^{15}$ while the planned Microwave Imaging Instrument (MWI) will offer $23 .{ }^{16}$ Sensitivity is also an issue and a recent study by Dongre et al. ${ }^{13}$ has indicated that maintaining and/or improving sensitivity as channel count increases is vital. In this paper, we report on an SFBS with TES readout as a technology for realizing a MW sounding instrument with several hundred channels and sky-noise limited performance. This would represent a disruptive advance in the field, allowing measurements of comparable performance to IR sounders under all sky conditions.

The chip spectrometer reported here is a demonstrator for an atmospheric temperature and humidity sounder (HYper-spectra Microwave Atmospheric Sounding: HYMAS), that is, being developed to operate at frequencies in the range of $v=45-190 \mathrm{GHz}^{13,17}$ The demonstrator was designed to cover the very important $\mathrm{O}_{2}$ absorption band at $v=50-60 \mathrm{GHz}$ for atmospheric temperature sounding. We believe, however, that our prototype designs and initial characterizations are already relevant across the broad band of scientifically important research areas described above.

In Sec. II, we give a brief overview of SFBSs and the particular features of the technology that make them attractive for this application. We will then describe the design of a set of devices to demonstrate the key technologies required for temperature sounding using the $\mathrm{O}_{2}$ absorption band at $50-60 \mathrm{GHz}$. In Sec. III, we describe the fabrication of the demonstrator chips and their assembly with electronics into a waveguide-coupled detector package. In Sec. IV, we report the first characterization of complete SFBS's with TES readout detecting at $40-65 \mathrm{GHz}$, considering the TES response calibration, measurements of overall detection efficiency, and measurements of filter response. Finally, we summarize the achievements and describe our future program and the pathway from this demonstrator to a full instrument.

\section{SUPERCONDUCTING ON-CHIP FILTER-BANK SPECTROMETERS}

In general, a filter-bank spectrometer uses a set of banddefining electrical filters to disperse the different spectral components of the input signal over a set of power detectors. In the case of an SFBS, the filters and detectors are implemented using microfabricated superconducting components and are integrated together on the same device substrate (the "chip"). The integration of the components on the same chip eliminates housings, mechanical interfaces, and losses between different components, helping to reduce the size of the system, while improving ruggedness and optical efficiency. In addition, it is easy to replicate a chip and, therefore, a whole spectrometer, making SFBSs an ideal technology for realizing spectroscopic imaging arrays.

Most mm- and sub-mm wave SFBSs that have been reported in the literature operate directly at the signal frequency, i.e., there is no frequency downconversion step. ${ }^{5,6}$ There are two main benefits of this approach, the first of which is miniaturization. The size of a distributed-element filter is intrinsically inversely proportional to the frequency of operation: the higher the frequency, the smaller the individual filters and the more channels that can be fitted on the chip. The second benefit is in terms of instantaneous observing bandwidth, which is in principle limited only by the feed for frequencies below the pair-breaking thresholds of the superconductors.

Operation at the signal frequency is made possible by (a) the availability of superconducting detectors for $\mathrm{mm}$ - and sub-mm wavelengths, and (b) the low intrinsic Ohmic loss of the superconductors. Critically, (b) allows filter channels with scientifically useful resolution to be realized at high frequencies. In the case of normal metals, increases in Ohmic loss with frequency and miniaturization of the components quickly degrade performance. As an example, the Ohmic losses in $\mathrm{Nb}$ microstrips line at sub-kelvin temperatures are expected to be negligible for frequencies up to $680 \mathrm{GHz}^{18}$ (the onset of pair-breaking). The use of ultra-low noise superconductor detector technology such as TESs and KIDs, (a), in principle allows for extremely high channel sensitivities.

The SFBS test chips described here were developed with the target application of satellite atmospheric sounding and against the specification given in Table I. Figure 1 shows the system level design of the chips. Each chip comprises a feeding structure that couples signal from a waveguide onto the chip in the range of 45$65 \mathrm{GHz}$, followed by a filter bank and a set of TES detectors. The chip is then housed in a test fixture that incorporates additional

TABLE I. Table of detector specifications for a baseline temperature sounder using the $\mathrm{O}_{2}$ absorption band. The background power loading and detector time constant have been calculated assuming the same optics and scan pattern as the MWI instrument. ${ }^{16}$ The required NEP gives near sky-noise limited performance under these conditions. The efficiency is referenced to the feed to the chip.

\begin{tabular}{lcc}
\hline \hline Parameter & Specification & Units \\
\hline Operating frequency range & $50-60$ & $\mathrm{GHz}$ \\
Filter resolution & $100-500$ & \\
Noise equivalent power & $\lesssim 10$ & $\mathrm{aW} / \sqrt{\mathrm{Hz}}$ \\
Detector time constant & 5 & $\mathrm{~ms}$ \\
Detector absorption efficiency & $50 \%$ & $\mathrm{fW}$ \\
Background power handling & 60 & $\mathrm{mK}$ \\
Operating temperature & $>300$ & \\
Number of channels & 35 & \\
\hline \hline
\end{tabular}




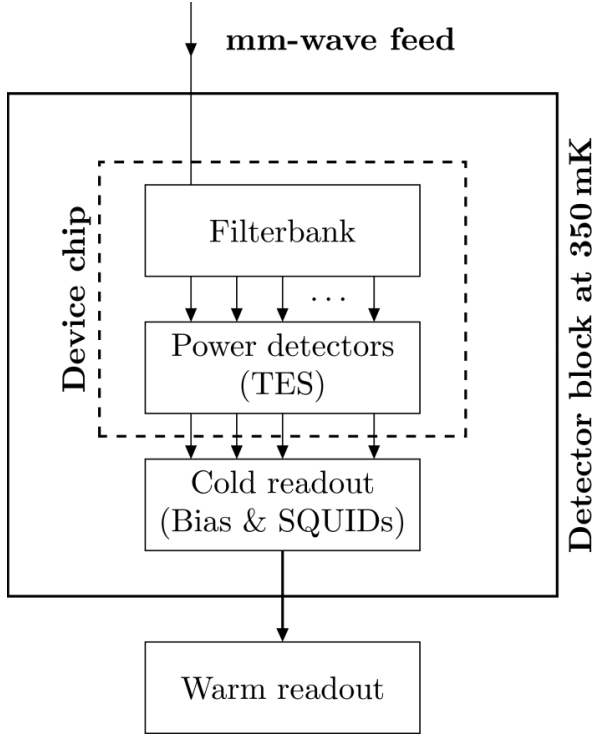

FIG. 1. Conceptual design of the test devices, also showing the architecture of a superconducting on-chip filter-bank spectrometer.

cold electronics and waveguide interfaces. In the sub-sections, we will describe the design of each of the components in detail.

Each of the test chips described has 12 detectors in total. This number was chosen for convenient characterization without multiplexed readout, but the architecture readily scales to higher channel count. In principle, the limiting factor is increasing attenuation on the feed line as more channels are added, but the losses on superconducting line are so low that other issues, such as readout capacity, are expected to be the main limit.

\section{A. Feed and transition}

$\mathrm{RF}$ signal input to the test devices is via a standard $1.88 \times$ $3.76 \mathrm{~mm}^{2}$ WR15 waveguide, allowing full coverage of the $\mathrm{O}_{2}$ band (WR15 has a recommended operating range of $50-75 \mathrm{GHz}$, singlemode operation from 39.875 to $79.750 \mathrm{GHz}$ ). The signal is coupled from the waveguide onto a $22.3 \Omega$ microstrip line on the chip through a radial probe transition, ${ }^{19}$ the design of which is shown in Fig. 2. A split block waveguide is used, the upper part of which has been rendered as transparent in the figure to allow the internal structure to be seen. A channel is machined through the waveguide wall at the split plane to accommodate a silicon beam extending from the chip, shown in green. This beam supports a fan-shaped radial probe, shown in blue, the apex of which connects to the upper conductor of the microstrip. The microstrip ground plane is shown in yellow. Not visible is an air channel under the beam, which raises the cut-off frequency of the waveguide modes of the loaded channel above the band of operation.

It is critical for performance that the ground plane is at the same potential as the waveguide/probe-channel walls at the probe apex; however, it is not straightforward to make a physical

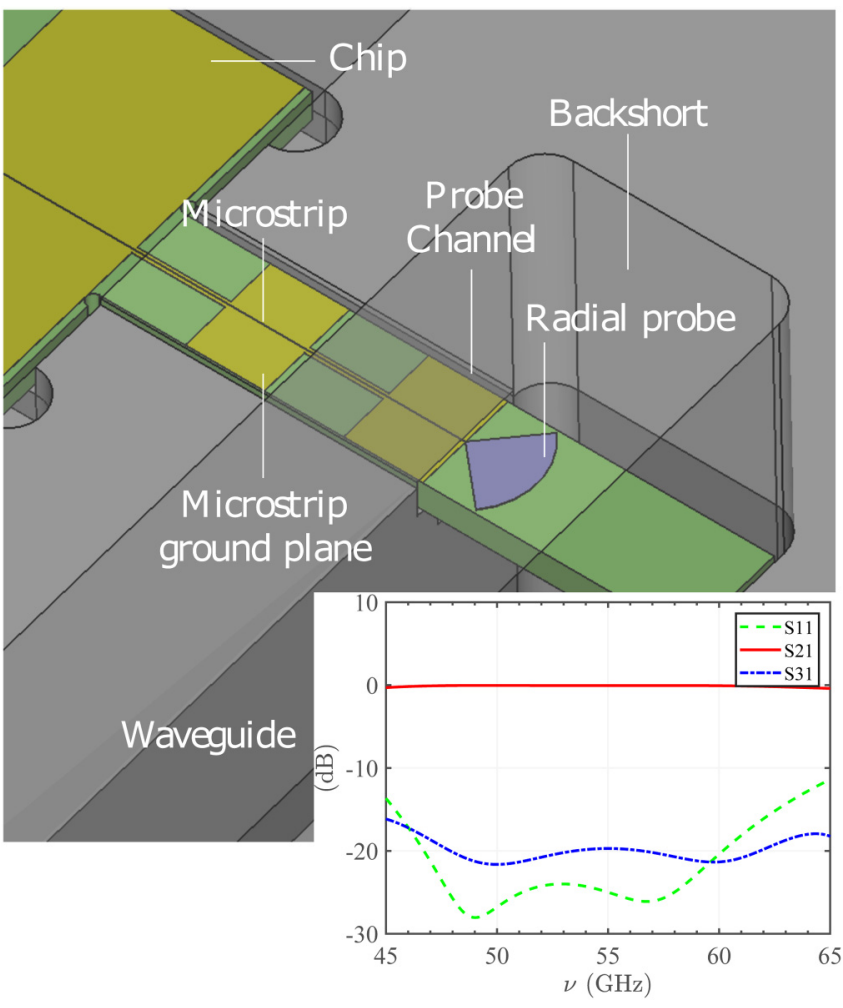

FIG. 2. Details of the waveguide to microstrip transition. The different components are described in the text. The inset shows the simulated scattering parameters, where ports 1, 2, and 3 are the waveguide input, microstrip output, and coupling to the chip cavity, respectively.

connection. The changes in ground plane width shown in Fig. 2 implement a stepped impedance filter in the ground plane/wall system to ensure a wideband short at the probe plane, assuming the ground plane is wire-bonded to the walls in the chip cavity. This filter also prevents power flow along the air channel due to the TEM mode.

The performance of the design was simulated using OpenEMS, a finite difference time domain (FDTD) solver. ${ }^{20}$ Insertion loss $\left(S_{21}\right)$, reflection loss $\left(S_{11}\right)$, and indirect leakage to the chip cavity $\left(S_{31}\right)$ as a function of frequency are shown in the inset of Fig. 2. As can be seen, the design achieves better than $-3 \mathrm{~dB}$ insertion loss over $45-65 \mathrm{GHz}$, corresponding to a fractional bandwidth of nearly $35 \%$.

\section{B. Filter bank}

The filter-bank architecture employed on the test chips is shown schematically in Fig. 3(a). It comprises ten half-wave resonator filters driven in parallel from a single microstrip feed line. Each of the filters comprises a length of microstrip line, the ends of which are connected by overlap capacitors to the feed and an output microstrip line. The output line is terminated by a matched 
mm-wave input microstrip feed line
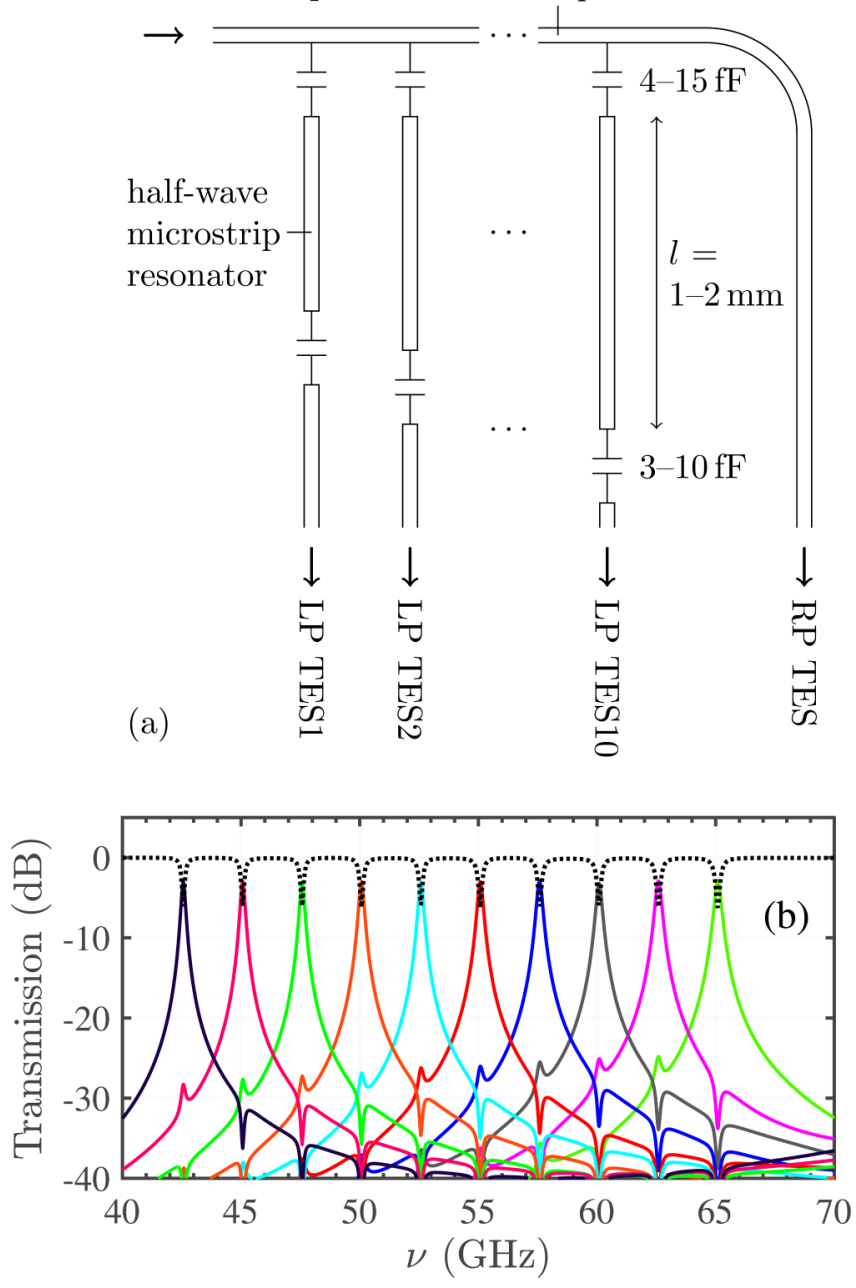

FIG. 3. Details of the filter-bank designs. (a) shows a schematic of the filter bank. (b) shows the power transmitted to individual channels for $\mathcal{R}=200$. The different channels are indicated by the different line colors. Transmission to the residual power detector terminating the feed is indicated by the black dotted line.

resistor that is closely thermally coupled to a TES bolometer. Both the termination resistor and TES are thermally isolated from the cold stage. The feed line itself is terminated on an 11th matched resistor coupled to a TES. This TES measures the power remaining after passing the filter bank and is subsequently described as the residual power detector. This TES was specifically designed to handle the expected higher incident power. A 12th detector on chip has no microwave connection and was used as a "dark" (i.e., nominally power-free) reference. A common superconducting microstrip design is used for all parts of the filter bank, consisting of a $\mathrm{Nb}$ ground plane, a $400-\mathrm{nm}$-thick $\mathrm{SiO}_{2}$ dielectric layer and a $3 \mu \mathrm{m}$ wide, $500 \mathrm{~nm}$ thick, $\mathrm{Nb}$ trace. The modeled characteristic impedance is $22.3 \Omega .^{18}$
The spectral characteristics of each filter are determined by the resonant behavior of the line section and the strength of the coupling to the feed and detector. For weak coupling, the line section behaves as an open-ended resonator and transmission peaks sharply at resonance.

Each filter channel is designed to have its own fundamental frequency $v_{0}$. For a filter of length $l$ and phase velocity $c, v_{0}$ is given by

$$
v_{0}=\frac{c}{2 l}
$$

Tuning over the range of $40-70 \mathrm{GHz}$ is achieved by values of $l$ in the range of 2.225-1.000 $\mathrm{mm}$. The channel resolution $\mathcal{R}=v_{0} / \Delta v$, where $\Delta v$ is the 3 - $\mathrm{dB}$ width.

The bandwidth of a filter and its peak-value of transmission are governed by losses in the resonator, as measured by the quality factor. Assuming power loss $P$ from the resonator when the stored energy is $U$, the total quality factor $Q_{t}$ of the resonator (and hence the resolution of the filter $\mathcal{R} \equiv Q_{\mathrm{t}}$ ) is defined by $Q_{\mathrm{t}}=2 \pi v_{0} U / P . P$ can be further decomposed into the sum of the power loss $P_{c, \text { in }}$ and $P_{c, \text { out }}$ to the input and output circuit, respectively (coupling losses) and the power dissipated $P_{\text {int }}$ in the resonator itself through Ohmic and dielectric loss (internal losses). We can then define additional quality factors $Q_{\text {int }}, Q_{c, \text { in }}$, and $Q_{c, \text { out }}$ by $Q_{n}=2 \pi v_{0} U / P_{n}$ These correspond to the $\mathrm{Q}$-factor of the resonator in the limit where the individual losses are dominant, and $Q_{t}^{-1}=Q_{\text {int }}^{-1}+Q_{c, \text { in }}^{-1}+Q_{c, \text { out }}^{-1}$. With these definitions, the power gain of the channel as a function of frequency $v$ can be shown to be

$$
G\left(v, v_{0}\right)=\frac{2 Q_{\mathrm{t}}^{2}}{Q_{\mathrm{c}, \text { in }} Q_{\mathrm{c}, \text { out }}} \frac{1}{1+4 Q_{\mathrm{t}}^{2}\left(v-v_{0}\right)^{2} / v_{0}^{2}} .
$$

$Q_{c, \text { in }}$ and $Q_{c, \text { out }}$ are controlled by the input and output coupling capacitors, respectively. For maximum transmission of power to the filter channel detector $(-3 \mathrm{~dB})$, we must engineer $Q_{c \text {,in }}=Q_{c \text {,out }}=Q_{c} \ll Q_{\text {int }}$, in which case $Q_{t}=Q_{c} / 2 \ll Q_{\text {int }}$. Therefore, the minimum achievable bandwidth is limited by $Q_{\text {int }}$. Under the same conditions, the power transmitted to the residual power detector is

$$
T\left(v, v_{0}\right)=1+\frac{Q_{t}}{Q_{c, \text { in }}} \frac{\left[Q_{\mathrm{t}} / Q_{\mathrm{c}, \text { in }}-2\right]}{1+4 Q_{\mathrm{t}}^{2}\left(v-v_{0}\right)^{2} / v_{0}^{2}},
$$

and the power is reduced by $6-\mathrm{dB}$ on resonance as seen from the dotted black line in Fig. 3(b). In practice, the presence of the coupling capacitors causes the measured center frequency $v_{0 \text {,meas }}$ of the filter to differ slightly from the resonant frequency of the open-ended line, as given by (1). The detuning is dependent on coupling strength and analysis of the perturbation of the circuit to first-order gives

$$
v_{0, \text { meas }} \approx\left(1-\frac{1+\sqrt{2}}{2 \sqrt{\pi \mathcal{R}}}\right) v_{0} .
$$

Ohmic loss is small in superconducting microstrip lines at low temperatures. Instead, experience with superconducting 
microresonators $<10 \mathrm{GHz}$ suggests dielectric loss due to two-level-systems will govern $Q_{\text {int. }}{ }^{21} \mathrm{~A}$ main aim of the prototype devices was to investigate the loss mechanisms active at millimeter wavelengths, although extrapolation from the low-frequency data suggest spectral resolutions of a few hundred should be easily achievable for the microstrip design used here, and higher may be achievable for alternatives such as coplanar waveguide.

We report on two of the four different filter-bank designs that were fabricated on the demonstrator devices. Three of these have identically spaced channels, designed to be $2.5 \mathrm{GHz}$ apart over the range of 42.5-65.00 GHz. The designs differ in spectral resolution of the channels, with target values of $\mathcal{R}=250,500$, and 1000 . These explore the achievable control of channel placement and resolution and provide experimental characterization of the millimeter-wave properties of the microstrip over a wide frequency range. However, the channels in these designs are widely spaced. The fourth design investigated an alternate possible mode of operation where the passbands of the filters overlap significantly, with nine $\mathcal{R}=500$ channels in the band $53-54 \mathrm{GHz}$. In this case, the spacing of the filters along the feed line becomes significant, as the filters interact with each other electrically. If the filters are arranged in order of decreasing frequency and placed approximately a quarter of a wavelength apart, part of the in-band signal scattered onto the feed by the filter is reflected back in phase from the next filter along, enhancing transmission to the detector. Consequently, the transmission of the filters can be increased by dense packing but at the expense of the shape of the response; we are investigating the implications for scientific performance. The modeled passbands for the widely spaced filter banks are shown in Fig. 3(b).

\section{TES design}

Electrothermal feedback (ETF) in a thermally isolated TES of resistance $R$, voltage-biased within its superconducting-normal resistive transition means that the TES self-regulates its temperature $T$ very close to $T_{c}$, the TES transition temperature. ${ }^{22}$ When the superconducting-normal resistive transition occurs in a very narrow temperature range $\alpha \gg 1$, where $\alpha=T(d R / d T) / R$ characterizes the transition sharpness. Provided $T, T_{c} \gtrsim 1.5 \mathrm{~T}_{\mathrm{b}}$ the bath temperature, the small-signal power-to-current responsivity $s_{I}$ is then given by $s_{I}=-1 /\left(I_{0} R_{0}\right)$. Here, $T_{0}, I_{0}$, and $R_{0}$ are the TES temperature, current, and resistance, respectively, at the operating point, and $R_{\mathrm{L}}$ is the load resistance. All are simple to evaluate from measurements of the TES and its known bias circuit parameters meaning that $s_{I}$ is straightforward to evaluate.

The TESs we report on were designed to operate from a bath temperature of $T_{\mathrm{b}} \simeq 300 \mathrm{mK}$ in order to be usable from a simple cryogenic cooling platform, to have a saturation power of order $P_{\text {sat }} \simeq 2 \mathrm{pW}$ to satisfy expected power loading, and a phononlimited noise equivalent power (NEP) of order $2 \mathrm{aW} / \sqrt{\mathrm{Hz}}$ to minimize detector NEP with respect to atmospheric noise (see Table I). TES design modeling indicates that the required performance should be achievable with a superconducting-normal transition temperature $T_{c} \sim 550 \rightarrow 650 \mathrm{mK}$. The detailed calculation depends on the value of the exponent $n$ that occurs in the calculation of the power flow from the TES to the heat bath: $P_{\mathrm{b}}=K_{\mathrm{b}}\left(T_{c}^{n}-T_{\mathrm{b}}^{n}\right)$, where $K_{\mathrm{b}}$ is a material dependent parameter that includes the geometry of the thermal link between the TES and bath. For ideal voltage bias $\left(R_{\mathrm{L}}=0\right)$, the saturation power is $P_{\text {sat }}=\left(1-R_{0} / R_{\mathrm{N}}\right) P_{\mathrm{b}}$ and $R_{\mathrm{N}}$ is the TES normal-state resistance. ${ }^{22}$ Under typical operating conditions, $R_{0} \sim 0.25 R_{\mathrm{N}}$. The thermal conductance to the bath $G_{\mathrm{b}}=d P_{\mathrm{b}} / d T_{c}$ determines the phonon-limited NEP, where $\mathrm{NEP}=\sqrt{4 k_{\mathrm{b}} \gamma G_{\mathrm{b}} T_{c}^{2}}, k_{\mathrm{b}}$ is Boltzmann's constant, and $\gamma \leq 1$ takes account of the temperature gradient across $G_{\mathrm{b}}$. Our previous work suggests that at the operating temperature and length scales $n \sim$ $1.5 \rightarrow 2.5$ should apply for 200 -nm-thick $\mathrm{SiN}_{\mathrm{x}}$ at these temperatures with length of $50-1000 \mu \mathrm{m}$. For the filter channels, thermal isolation of the TES was formed from four 200-nm-thick $\mathrm{SiN}_{\mathrm{x}}$ legs each of length $500 \mu \mathrm{m}$, three of width $1.5 \mu \mathrm{m}$, and one of $4 \mu \mathrm{m}$ to carry the microstrip. The residual power detector had support legs of length $50 \mu \mathrm{m}$ and the same widths as for the filter channels.

\section{FABRICATION AND ASSEMBLY}

The detector chips were fabricated on $225 \mu \mathrm{m}$ thick Si wafers coated with a dielectric bilayer comprising 200-nm-thick low-stress $\mathrm{SiN}_{\mathrm{x}}$ and an additional $50 \mathrm{~nm} \mathrm{SiO}_{2}$ etch stop. After Deep Reactive Ion Etching (DRIE), the $\mathrm{SiN}_{\mathrm{x}}, \mathrm{SiO}_{2}$ films formed the thermal isolation structure. The detectors were made by sputtering metal and dielectric thin films in ultra-high vacuum. The superconducting microstrip was formed from a DC-sputtered $150 \mathrm{~nm}$-thick $\mathrm{Nb}$ ground plane with $400 \mathrm{~nm}$ amorphous silicon dioxide $\left(\mathrm{SiO}_{2}\right)$ dielectric deposited by RF-sputtering. A second deposition of $\mathrm{Nb}$ $500 \mathrm{~nm}$-thick $3 \mu \mathrm{m}$-wide completed the microstrip. The ground plane $\mathrm{Nb}$ also formed the TES wiring. The measured $\mathrm{Nb}$ residual resistance ratio (RRR) was 2.7-3.0. Coupling capacitors were made from $\mathrm{SiO}_{2}$ and $\mathrm{Nb}$. Thin-film $\mathrm{AuCu}$ resistors terminate the microstrip on the TES island. The TESs were fabricated from TiAl bilayers with the $\mathrm{Ti}$ and $\mathrm{Al}$ thicknesses of $150 \mathrm{~nm}$ and $40 \mathrm{~nm}$, respectively, calculated to target a superconducting transition temperature of $600-650 \mathrm{mK}^{23}$ DRIE removed the Si under the TES such that the $\mathrm{SiN}_{\mathrm{x}}$ island and legs provide the necessary thermal isolation. The DRIE also released the individual chips from the host wafer and defined the chip shape.

Figure 4(a) shows an image of the residual power detector prior to DRIE of the Si. The $4 \mu \mathrm{m}$-wide leg supporting the microstrip is indicated in the lower left-hand region of the image. The $3 \times 35 \mu \mathrm{m}^{2} \mathrm{AuCu}$ termination can be seen. Figure 4(b) shows a detail of the filter bank. The insets in the upper quadrants to the left and right show detailed images of the T-section coupling capacitors to the through line and the inline coupler to the TES detectors, respectively. In all cases, the microstrip was $3 \mu \mathrm{m}$ wide. Filter channels 4 and 8 are indicated. Filter lengths are determined by the line length between the T-section and inline capacitors. The lengths increase with channel number (left-to-right in the image) and correspondingly $v_{0}$ reduces. Figure 4 (c) shows a composite high resolution photograph of a completed detector chip after DRIE. The component structures, waveguide probe, filter bank, TES detectors, and superconducting contact pads are indicated. The individual filter channels are also visible.

Figure 5 shows an image of a completed chip mounted in a detector enclosure. The probe can be seen extending into the lower half of the split waveguide. Al wirebonds connect the chip ground plane to the enclosure and on-chip electrical wiring to the 

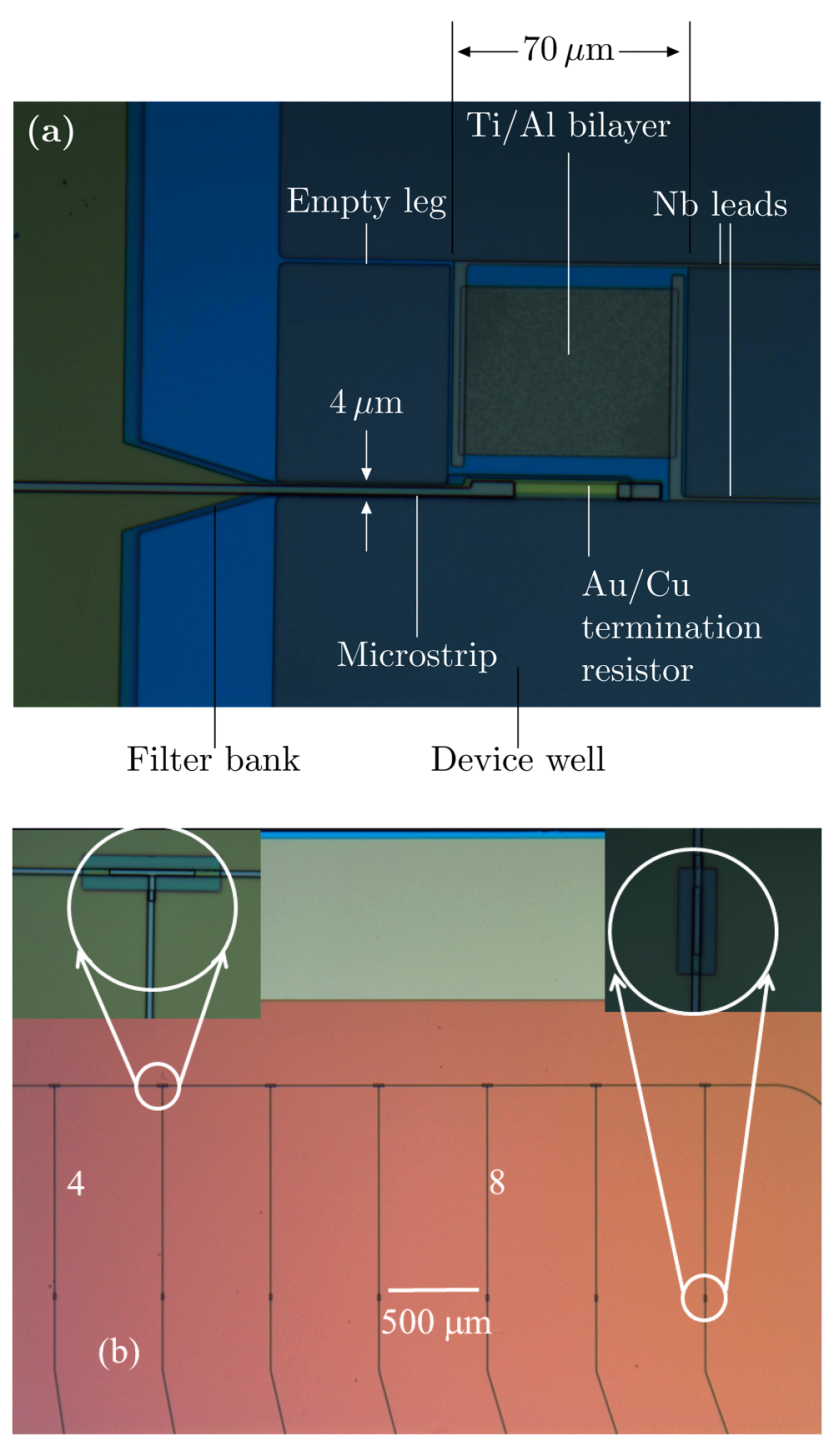

Waveguide probe Microstrip Filterbank

Wafer tab

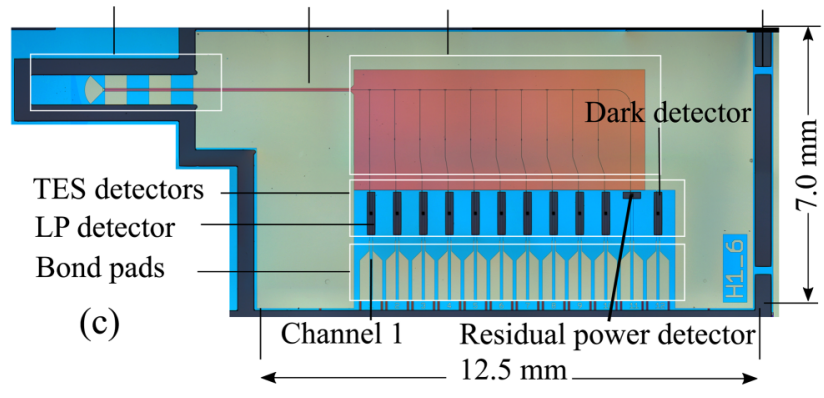

FIG. 4. (a) shows a high resolution optical image of a residual power detector. (b) shows a high resolution optical image of the filter bank. The expanded images in the upper left and right quadrants show details of a T-section and inline capacitive coupler. (c) is an optical image of a complete demonstrator chip.

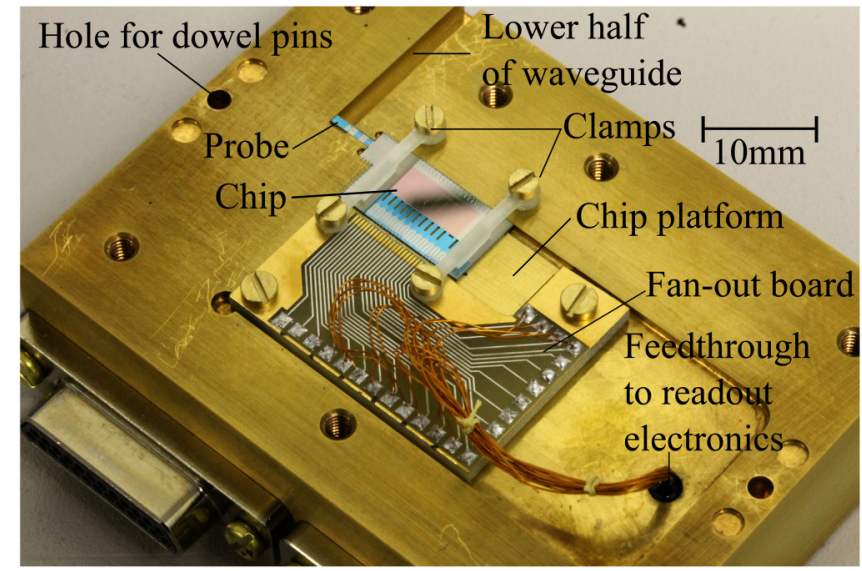

FIG. 5. Photograph of the assembled lower half of the detector enclosure. The upper part forms a light-tight enclosure and completes the upper section of the split waveguide.

superconducting fan-out wiring. Superconducting $\mathrm{NbTi}$ wires connect through a light-tight feedthrough into the electronics enclosure on the back of the detector cavity. The low-noise twostage SQUIDs used here were provided by Physikalisch-Technische Bundesanstalt (PTB). The upper section of the detector enclosure completed the upper section of the waveguide and ensured that the package was light-tight. Completed detector packages were cooled using two separate cryogen-cooled ADRs both giving a base temperature $T_{\mathrm{b}} \lesssim 100 \mathrm{mK}$.

Figure 6(a) shows a schematic of the blackbody power calibration scheme. A matched $50 \Omega$ resistive load (Fairview Microwave

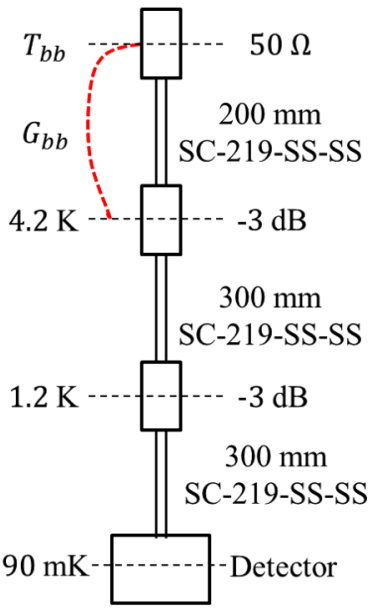

(a)

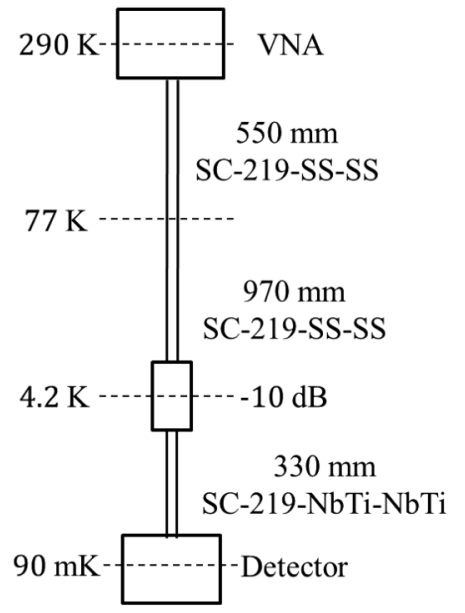

(b)
FIG. 6. Schematics of the measurement scheme. (a) For the blackbody power calibration. (b) For the measurement of filter spectral response. 
SKU:ST6510 ${ }^{24}$ terminated a length of Coax Co. Ltd SC-219/ 50-SS-SS coaxial line. ${ }^{25}$ The load was mounted on a thermally isolated $\mathrm{Cu}$ plate within the cryostat $4 \mathrm{~K}$ vacuum space. This termination-resistor stage was equipped with a calibrated LakeShore $\mathrm{RuOx}$ thermometer ${ }^{26}$ and resistive heater and was connected to the refrigerator $4 \mathrm{~K}$ stage by a length of copper wire $(l=20 \mathrm{~cm}, r=250 \mu \mathrm{m})$ that determined the dominant stage time constant which was estimated at $\sim 20 \mathrm{~ms}$. Furthermore, SC-219 connected the termination resistor to a $3-\mathrm{dB}$ attenuator (Fairview Microwave SA6510-03) mounted on the $4 \mathrm{~K}$ stage itself, to a further attenuator mounted on the $1 \mathrm{~K}$ stage, in order to minimize heat-loading to the cold stage by heat-sinking the inner coax conductor and then to the WR-15 coax-waveguide transition (Fairview Microwave SKU:15AC206), waveguide probe, and detector chip. The total coax length was $800 \mathrm{~mm}$. Results of these measurements are reported in Sec. V.

The spectral response of the filters was measured using a continuous wave (CW) source. For these tests, a different cryostat was used, but it again comprised a two-stage $(1 \mathrm{~K} / 50 \mathrm{mK})$ ADR launched from a $4 \mathrm{~K}$ plate cooled by liquid cryogens. A different set of cold electronics with nine single-stage SQUIDs provided by PTB was used that allowed simultaneous readout of nine devices.

Figure 6(b) shows a schematic of the scheme. A Rohde and Schwarz ZVA-67 VNA capable of measurements up to $67 \mathrm{GHz}$ was used as a frequency-tunable, power-leveled, CW source and the signal coupled down to the detector block on coaxial cable. A KMCO KPC185FF HA hermetic feedthrough was used to enter the cryostat, and then $1520 \mathrm{~mm}$ of Coax Co. Ltd SC-219/ 50-SS-SS coaxial cable was used for the connection between room temperature and the $4 \mathrm{~K}$ plate, anchoring on the liquid nitrogen tank, liquid helium tank, and finally, the $4 \mathrm{~K}$ plate itself. The final connection from $4 \mathrm{~K}$ to the waveguide adapter on the test block was made using $330 \mathrm{~mm}$ of Coax Co. Ltd SC-219/50-NbTi-NbTi superconducting cable for thermal isolation. A $10 \mathrm{~dB}$ attenuator (Fairview Microwave SA6510-10) was inserted in line at the $4 \mathrm{~K}$ plate to provide thermal anchoring between the inner and outer conductors of the coaxial cable and isolation of the detectors chip from short-wavelength power loading. All connections were made with $1.85 \mathrm{~mm}$ connectors to allow mode free operation up to $65 \mathrm{GHz}$. Measurements indicated the expected total attenuation of order $68 \mathrm{~dB}$ between room temperature and the WR-15 waveguide flange. In operation, the frequency of the $\mathrm{CW}$ source was stepped through a series of values and the output of the devices logged in the dwell time between transitions, exploiting the normal mode of operation of the VNA. Results of these measurements are reported in Sec. VI.

Two chips were characterized in this series of tests. Both chips were fabricated on the same wafer but have different designed filter resolving powers. Chip 1 has filters designed with $\mathcal{R}=500$ was used for the blackbody measurements with measurements on filter channels 4-6,11 (the residual power detector), and 12 (the dark detector). Chip 2 with $\mathcal{R}=200$ was used for the spectral measurements and we report measurements on channels $3-8,10$, and 12 . Both chips were designed to cover the 42.5$65 \mathrm{GHz}$ range.

\section{RESULTS}

\section{A. TES characteristics}

Table II shows values for the DC characteristics of five channels from Chip 1 calculated from measurements of the current-voltage response of the devices with bath temperature. $T_{c}$ was close-to but somewhat lower than designed, and $R_{\mathrm{N}}$ was higher. The critical current density was also reduced from our previously measured values even for pure $\mathrm{Ti}$ films. This may indicate that these TESs demonstrate the thin-film interdiffusion effects between $\mathrm{Ti}-\mathrm{Nb}$ thin-film interfaces recently reported by Yefremenko et al. ${ }^{27}$ The superconducting-normal resistive transition with $T_{\mathrm{b}}$, as inferred from measurements of the TES Joule power dissipation as a function of bias voltage, appeared somewhat broad. Hence, $G_{\mathrm{b}}$ was determined from the power dissipated at constant TES resistance $R_{0}=0.25 R_{N}$, close to the bias voltage used for power measurements. The exponent in the power flow was $n=2.0 \pm 0.1$ for all TESs. The calculated phonon-limited NEP was $6.1 \pm 0.1 \mathrm{aW} / \sqrt{\mathrm{H} z}$ assuming a noise-modifier $\gamma=0.7$, extrapolating to $9.2 \pm 0.1 \mathrm{aW} / \sqrt{\mathrm{Hz}}$ at the target $T_{c}$ of $550 \mathrm{mK}$.

\section{B. TES ETF parameters and power-to-current sensitivity estimate}

For a TES modeled as single heat capacity $C$ with conductance to the bath $G_{\mathrm{b}}$, the measured impedance $Z(f)$ is given by ${ }^{22}$

$$
Z(f)=R_{\mathrm{L}}+j 2 \pi f L_{\mathrm{in}}+Z_{\mathrm{TES}}(f),
$$

where $R_{\mathrm{L}}$ is the load resistance, $L_{\text {in }}$ is the input inductance of the SQUID plus any stray inductance, $Z_{\mathrm{TES}}(f)$ is the TES impedance, and $f$ is the measurement frequency. The TES impedance is given by

$$
Z_{\mathrm{TES}}(f)=R_{0}(1+\beta)+\frac{R_{0} L_{I}}{1-L_{I}} \frac{2+\beta}{1+2 \pi f \tau_{I}},
$$

where $L_{I}=P_{0} \alpha / G_{\mathrm{b}} T_{0} . P_{0}=I_{0} R_{0}^{2}$ is the Joule power at the bias point, $T_{0} \simeq T_{c}$ is the operating temperature, $\tau_{I}=C /\left[G_{\mathrm{b}}\left(1-L_{I}\right)\right]$, and $\alpha=T(d R / d T) / R$ characterizes the sharpness of the resistive transition at the bias point. $\beta=I(d R / d I) / R$ measures the sensitivity of the transition to changes in current $I$. At measurement frequencies much higher than the reciprocal of the

TABLE II. Summary of DC TES characteristics for Chip 1.

\begin{tabular}{lccccc}
\hline \hline Channel & $R_{\mathrm{N}}(\Omega)$ & $T_{c}(\mathrm{mK})$ & $n$ & $G_{\mathrm{b}}(\mathrm{pW} / \mathrm{K})$ & Notes \\
\hline 4 & 2.6 & 457 & $2.0 \pm 0.1$ & 4.7 & Filter \\
5 & 2.5 & 452 & $2.0 \pm 0.1$ & 4.75 & Filter \\
6 & 2.4 & 454 & $2.0 \pm 0.1$ & 4.8 & Filter \\
12 & 2.4 & 455 & $2.0 \pm 0.1$ & 4.7 & Dark \\
11 & 2.4 & 459 & $2.0 \pm 0.1$ & 65 & Residual \\
& & & & & power \\
\hline \hline
\end{tabular}


effective TES time (here taken to be $f \geqslant 5 \mathrm{kHz}$ ), $\operatorname{Re}(Z(f))=R_{0}(1+\beta)$, and so $\beta$ can be determined with minimal measured parameters. At low frequencies, here taken to be $f \lesssim 5 \mathrm{~Hz}, \operatorname{Re}(Z(f))=R_{\mathrm{L}}+R_{0}(1+\beta)-\left(R_{0} L_{I}(2+\beta)\right) /\left(L_{I}-1\right)$ so that $L_{I}$ and hence $\alpha$ can be found. The low-frequency power-to-current responsivity is given by

$$
s_{I}(0)=-\frac{1}{I_{0} R_{0}}\left[\frac{R_{\mathrm{L}}+R_{0}(1+\beta)}{R_{0} L_{I}}+1-\frac{R_{\mathrm{L}}}{R_{0}}\right]^{-1} .
$$

For a TES with good voltage bias $R_{\mathrm{L}} \ll R_{0}$, and a sharp transition $\alpha, L_{I} \gg 1$, then provided $\beta \ll L_{I}-1, s_{I}(0)=-1 /\left(I_{0} R_{0}\right)$ and the power-to-current responsivity is straightforward to calculate. For the TiAl TES reported here the measured powervoltage response suggested a fairly broad superconductingnormal transition. Here, we use the full expression given by Eq. (7) with $s_{I}(0)=-k_{s} /\left(I_{0} R_{0}\right)$ and $k_{s}$ the calculated value of the reciprocal within the brackets.

The inset of Fig. 7 shows the measured real and imaginary parts (blue and green dots, respectively) of the impedance of the residual power detector, channel 11 . The solid red and dashed black lines show the modeled impedance. Table III shows the derived ETF parameters and heat capacity. The main figure shows the measured normalized TES current response (blue dots) to a small change in TES bias voltage (i.e., a small modulation of instantaneous Joule power), and (red line) the calculated response using the parameters determined in modeling the impedance. No additional parameters were used.

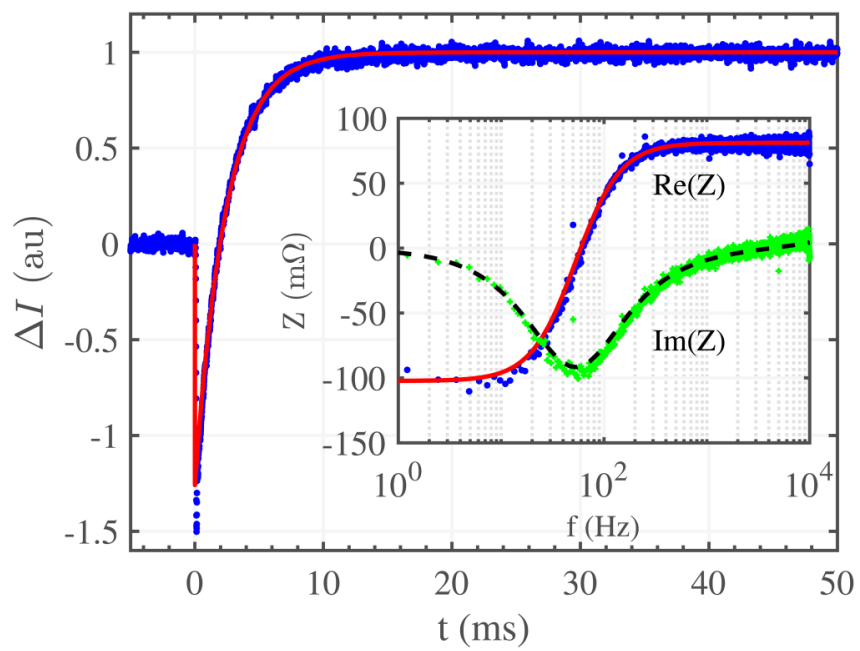

FIG. 7. The inset shows the measured real and imaginary parts (blue and green dots, respectively) of the impedance of the residual power detector (channel 11). The solid red and dashed black lines are the modeled values (real and imaginary, respectively) using the parameters given in Table III. The main plot shows the measured (blue dots) and calculated (red line) response of the TES current to a small step change in the bias voltage for the same channel with no additional parameters.
TABLE III. Summary of calculated ETF parameters.

\begin{tabular}{lrcccc}
\hline \hline Channel & $\alpha$ & $\beta$ & $C(\mathrm{f} / \mathrm{K})$ & $k_{s}$ & Notes \\
\hline 4 & 66 & 1.3 & 220 & 0.77 & Filter \\
5 & 77 & 2.9 & 200 & 0.74 & Filter \\
6 & 114 & 4.4 & 200 & 0.80 & Filter \\
12 & 128 & 1.3 & 180 & 0.88 & Dark \\
11 & 114 & 1.9 & 390 & 0.66 & Residual power \\
\hline \hline
\end{tabular}

The inset of Fig. 8 shows the measured real and imaginary parts (blue and green dots, respectively) of the impedance of the dark detector, channel 12. The solid red and dashed black lines show the modeled impedance. Table III shows the derived ETF parameters and heat capacity. The main figure shows the measured normalized TES current response (blue dots) to a small change in TES bias voltage (i.e., a small modulation of instantaneous Joule power), and (red line) the calculated response using the parameters determined in modeling the impedance. As in the modeling shown in Fig. 7, no additional parameters were used. Comparable correspondence between measured impedance and current step response using impedance-derived ETF parameters and heat capacity was found for all measured channels.

Derived values for $\alpha, \beta$, the heat capacity, and $k_{s}$ for Chip 1 used in the modeling of Figs. 7 and 8 are given in Table III. We see that $\alpha$ is low compared to our MoAu TESs, where $\beta \sim 0.05 \alpha{ }^{28}$ and the responsivity is reduced from the high- $\alpha$ value $k_{s}=1$. The

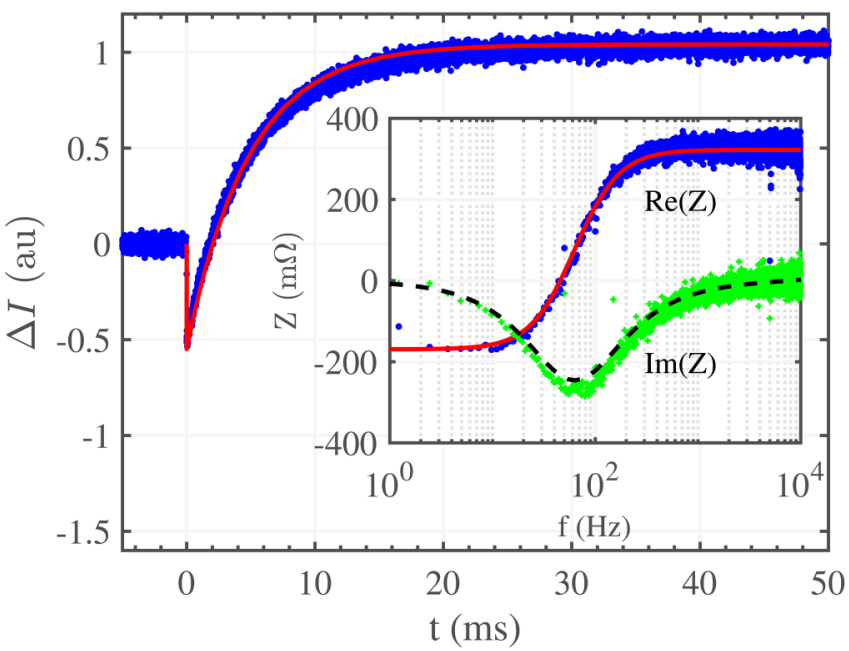

FIG. 8. The inset shows the measured real and imaginary parts (blue and green dots, respectively) of the impedance of the dark detector (channel 12). The solid red and dashed black lines are the modeled values (real and imaginary, respectively) using the parameters given in Table III. The main plot shows the measured (blue dots) and calculated (red line) response of the TES current to a small step change in the bias voltage for the same channel without additional parameters. 
large variation in $\alpha$ may-well be a result of the same materials problem discussed in Sec. IV A.

\section{POWER CALIBRATION}

\section{A. Available in-band power}

We assume that the blackbody load, coax connection, 3-dB attenuators, $1.85 \mathrm{~mm}$ connectors, waveguide, probe, and matched termination load behave as an ideal wideband single-mode blackbody source at temperature $T_{\mathrm{bb}}$. The optical bandwidth is determined by the components in the source rather than the detector itself. The lower band edge is determined by the cut-off frequency of the lowest order mode in the WR15 waveguide section at $v_{\min }=39.9 \mathrm{GHz}$. The upper cut-off frequency is determined by the $1.85 \mathrm{~mm}$ ('V-') connectors in the coaxial section ( 8 being used), which have a low-pass response with a $3-\mathrm{dB}$ point at $65 \mathrm{GHz}$. Raising the lower edge to account for the width of the cut-on region and performance of the waveguide transitions, the estimated total bandwidth is $45-65 \mathrm{GHz}$.

Fitting a model to the manufacturer's data, we find that loss in the coax can be described by $\alpha_{\text {coax }}(l)[\mathrm{dB}]=0.2648 l[\mathrm{~mm}] / \sqrt{v[\mathrm{GHz}]}$, where $l$ is the total line length. Additional losses arise from the $3-\mathrm{dB}$ attenuators that form the coax heat-sinks ( 2 being used), and the $1.85 \mathrm{~mm}$ connectors $(8$ in total), each contributing an additional loss of $\alpha_{\mathrm{c}}=0.223 \mathrm{~dB}$. The total attenuation $\alpha_{l}(v)$ (in $\mathrm{dB}$ ) is then

$$
\alpha_{l}(v)=\alpha_{\text {coax }}(l)+6+8 \alpha_{\mathrm{c}} .
$$

The change in available power at the probe is $\Delta P_{\max }\left(T_{b b}\right)=\left(P_{0}\left(T_{\mathrm{bb}}\right)-P_{0}\left(T_{0}\right)\right) 10^{-\alpha_{l}(v) / 10}$, with $T_{0}$ the lowest operating temperature for the blackbody and

$$
P_{0}(T)=\int_{v_{\min }}^{v_{\max }} h v n(v, T) d v,
$$

where $n(v, T)=\left[\exp \left(h v /\left(k_{\mathrm{b}} T\right)-1\right)\right]^{-1}$ is the Bose distribution and $h$ is Planck's constant.

To calculate the power transmission to the residual power detector, we assume each of the filter channels of center frequency $v_{0, i}$ (where $i$ identifies the channel and $i \in 1, \ldots, 10$ ) taps its maximum fraction of the incident power given by Eq. (2) and the transmitted power is given by Eq. (3), with $6-\mathrm{dB}$ attenuation at the residual power detector due to each filter at its resonant frequency. The total available power at the residual power detector $P_{r}\left(T_{\mathrm{bb}}\right)$ is

$$
P_{r}(T)=\eta \int_{v_{\min }}^{v_{\max }} h v n(v, T) \prod_{i} T_{i}\left(v, v_{0, i}\right) 10^{-\alpha_{l}(v) / 10} d v,
$$

where $\Pi$ is the product and $\eta$ is the overall efficiency referenced to the input to the probe.

\section{B. Coupled power measurement}

Measurements of the detection efficiency were made on Chip 1 with the cold stage temperature maintained at $90 \mathrm{mK}$ using a PID feedback loop to regulate the ADR base temperature. Under typical (unloaded) conditions, the cold stage temperature is constant at $T_{\mathrm{b}}= \pm 100 \mu \mathrm{K} . T_{\mathrm{bb}}$ was increased by stepping the blackbody heater current at $2 \mathrm{~Hz}$ with the change in measured TES current and $T_{\mathrm{bb}}$ digitized at $2 \mathrm{kHz}$. Changes in detected power in both the residual power and dark detectors, as $T_{\mathrm{bb}}$ was increased, were calculated from the change in their respective measured currents assuming the simple power-to-current responsivity, $s_{I}(0)=-1 /\left(I_{0} R_{0}\right)$, with the TES operating points calculated from measured and known electrical circuit parameters. At the highest $T_{\mathrm{bb}}$ used, $\sim 10 \mathrm{~K}, T_{\mathrm{b}}$ increased by $\sim 1 \mathrm{mK}$ suggesting power loading onto the cold stage. Under the same blackbody loading, and even after subtracting the expected dark detector power response due to $T_{\mathrm{b}}, \Delta P_{\mathrm{dark}}=G_{\mathrm{b}, \mathrm{dark}} \Delta T_{\mathrm{b}}$, the dark detector indicated a current (hence power) response that we interpret here as additional incident power. We modeled this residual response as a change in the temperature of the Si chip itself $T_{\text {chip }}$, finding an increase of order $3 \mathrm{mK}$ at the highest $T_{\mathrm{bb}}$. The modeled chip response $\Delta T_{\text {chip }}$ closely followed a quadratic response with $\Delta T_{\mathrm{bb}}$. Although the origins of this additional power loading could not be determined in this work, it may have arisen from residual short-wavelength loading from the blackbody source. At $10 \mathrm{~K}$, the peak in the multimode blackbody spectrum is around $1 \mathrm{THz}$ implying that there may be a significant available detectable power at high frequencies. Even a very small fraction of this power would be sufficient to produce the inferred chip heating. Changes in detected power for the residual power detector with $T_{\mathrm{bb}}$ were accordingly reduced to include the effect of both the measured $\Delta T_{\mathrm{b}}$ and the modeled $\Delta T_{\text {chip }}$.

Figure 9 shows the change in detected power $\Delta P_{r}$ as a function of $\Delta T_{\mathrm{bb}}$, for $T_{\mathrm{bb}}$ in the range $4.2-9.4 \mathrm{~K}$. The power response is close

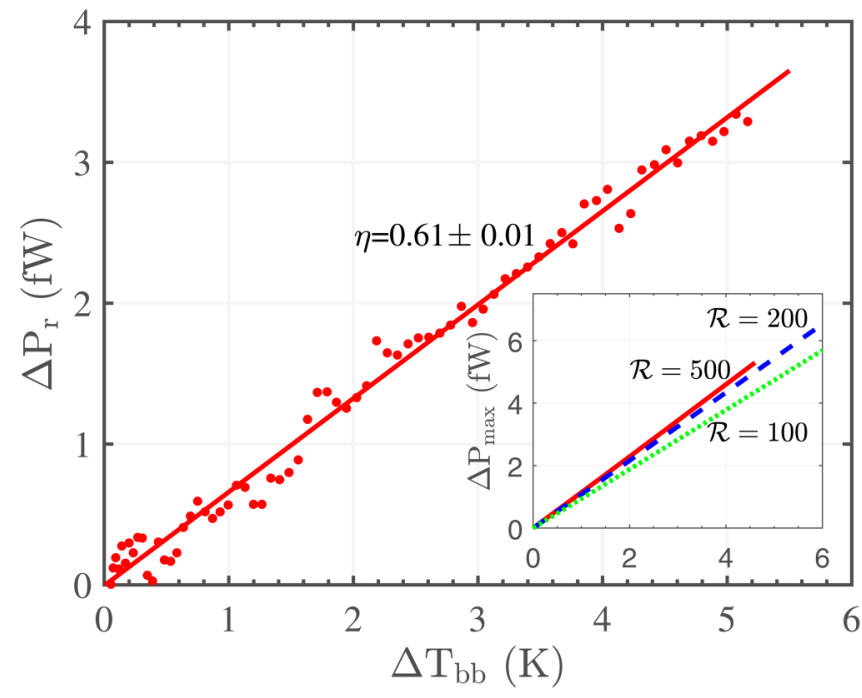

FIG. 9. Power detected for the residual power detector as a function of change in blackbody temperature. The inset shows the maximum available power $P_{\max }$ for three values of the filter-bank resolution. $\mathcal{R}=500$ is the designed resolution. The indicated efficiency $\eta$ assumes $\mathcal{R}=200$ and was calculated with the maximum TES responsivity $s_{i}=-1 /\left(I_{0} R_{0}\right)$. 
to linear with temperature (the correlation coefficient $R_{\text {cor }}=0.98$ ). The inset shows the maximum available power $P_{\max }$ calculated for three values of the filter resolution $\mathcal{R}_{i}=100,200$, and 500-the designed value. The residual power increases as $\mathcal{R}_{i}$ increases. As discussed in Sec. VI, the designed value may represent an overestimate of the achieved $\mathcal{R}$ for the measured chip. Assuming $\mathcal{R}=200$, we estimate an efficiency of $0.61_{-0.03}^{+0.01}$ and the lower error is calculated assuming the designed filter resolution $\mathcal{R}=500$. The measured power and hence efficiency estimate were calculated assuming the maximum power-to-current responsivity $s_{i}=-1 /\left(I_{0} R_{0}\right)$. From the measured response of the residual power detector described in Sec. IV B and Table III, we see that this overestimates the sensitivity by a factor $1 / k_{s} \simeq 1.5$. Taking account of this correction, our final estimate of overall efficiency referred to the input to the probe is increased to $\eta=0.91_{-0.05}^{+0.015}$.

\section{FILTER SPECTRAL RESPONSE}

Measurements of the spectral response were made on Chip 2. Figure 10 shows(dots) the normalized power detected and (solid lines) the modeled response using Eq. (2) (i.e., a Lorentzian) for filter channels 3-8 and 10 as labeled. Colors and annotation identify the individual channels. The individual filter responses $\mathcal{R}_{i}$ and $v_{0, i}$ are shown in Table IV along with the nominal design filter center frequencies. Errors in $v_{0, i}$ were calculated from the fit to the data. The frequency sampling of channel 10 was insufficient to determine the filter response. For this channel, the model fit shown was calculated assuming $\mathcal{R}_{10}=90.2$ (the mean of the other channels) and is plotted only as a guide to the eye. For this series of measurements, within measurement noise, there was no observable response in the dark channel and we estimate $\Delta P_{\text {dark }}<100 \mathrm{aW}$. The absence of any detectable dark response using a power-leveled, narrow-band, source operating at a fixed temperature of $\sim 300 \mathrm{~K}-$

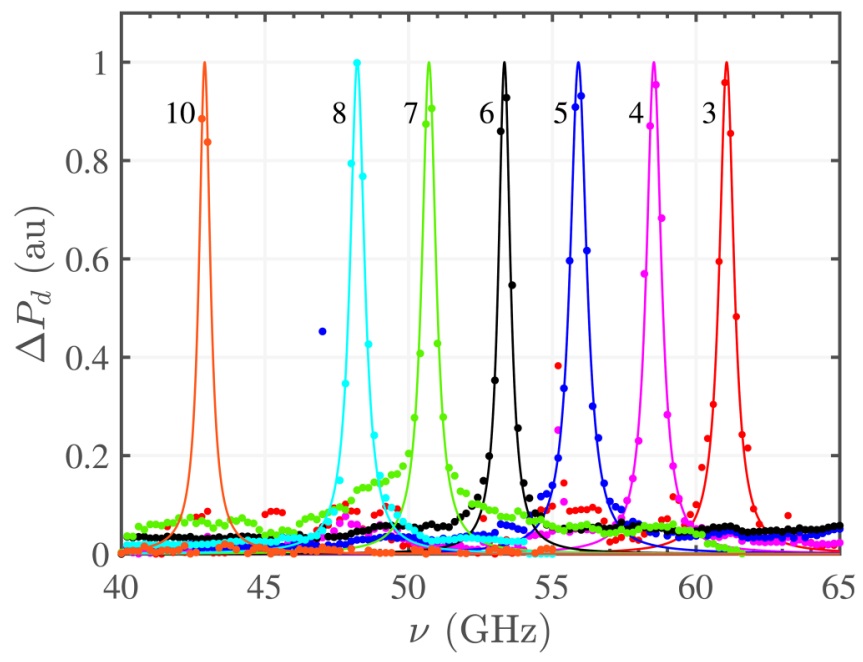

FIG. 10. Normalized power detected for filter channels 3-8 and 10 for Chip 2 (dots) and the calculated filter profiles (full lines). Colors and annotation identify the individual channels.
TABLE IV. Summary of designed and measured filter channel characteristics.

\begin{tabular}{lccr}
\hline \hline Channel & $\begin{array}{c}v_{\text {design }} \\
(\mathrm{GHz})\end{array}$ & $\begin{array}{c}v_{0, i} \\
(\mathrm{GHz})\end{array}$ & \multicolumn{1}{c}{$\mathcal{R}_{i}$} \\
\hline 3 & 60.0 & $61.06 \pm 0.013$ & $99.0 \pm 6.5$ \\
4 & 57.5 & $58.53 \pm 0.008$ & $92.8 \pm 3.4$ \\
5 & 55.0 & $55.90 \pm 0.013$ & $79.3 \pm 4.2$ \\
6 & 52.2 & $53.33 \pm 0.003$ & $106.5 \pm 2.0$ \\
7 & 50.0 & $50.71 \pm 0.010$ & $85.3 \pm 5.5$ \\
8 & 47.5 & $48.21 \pm 0.005$ & $78.4 \pm 1.9$ \\
10 & 42.5 & $42.89 \pm 0.001$ & \\
\hline \hline
\end{tabular}

in which case we might expect no, or only very small changes in additional loading-gives weight to our interpretation and analysis that the blackbody and efficiency measurements should indeed be corrected for heating from a broad-band, temperature modulated source. In those measurements, the dark detector showed an unambiguous response. The shapes of the individual filter responses were close to the expected Lorentzian as shown in more detail in Fig. 11 for channels 3 and 5 .

Figure 12 shows the measured filter center frequencies $v_{0}$ as a function of reciprocal filter length $l$. The correlation is close to unity $\left(R_{\text {cor }}=0.9998\right)$ as predicted by Eqs. (1) and (4), demonstrating the precision with which it is possible to position the individual filter channels. From the calculated regression line $v_{0}(\mathrm{GHz})=(66.15 \pm 0.20) / l(\mathrm{~mm})-(1.14 \pm 016)$, and assuming a modest lithographic precision of $\pm 1 \mu \mathrm{m}$ for the fabrication process, we calculate $\delta v= \pm 37 \mathrm{MHz}$ for a $50 \mathrm{GHz}$ filter, or $\delta v / v=0.0007$. The finite value for the intercept is unexpected and is an area for future investigation. Uncertainties in parameters such as the realized coupling capacitance and the exact permittivity of the dielectric constant are expected, but from Eqs. (1) and (4),

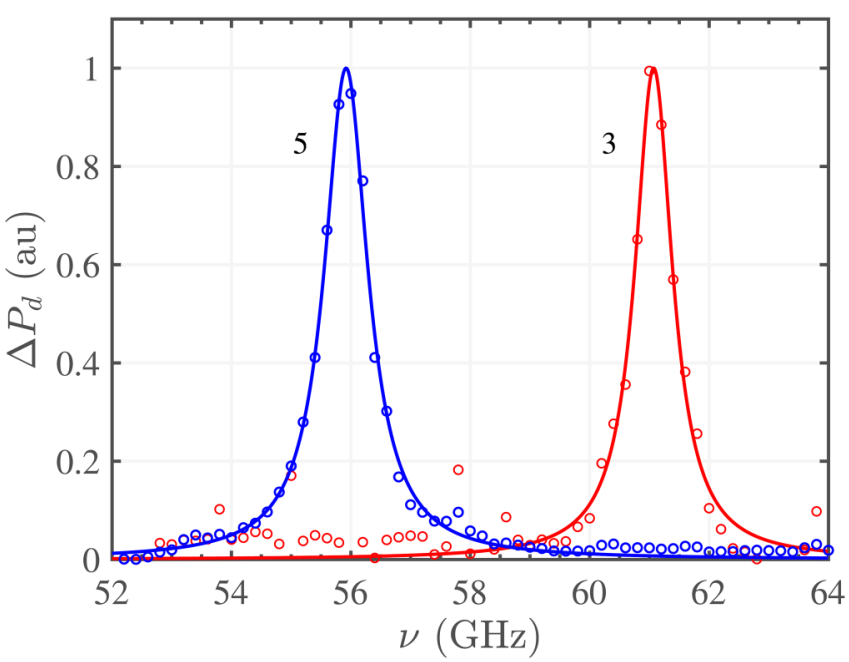

FIG. 11. Power detected from filter channels 3 and 5 with Lorentzian fits. 


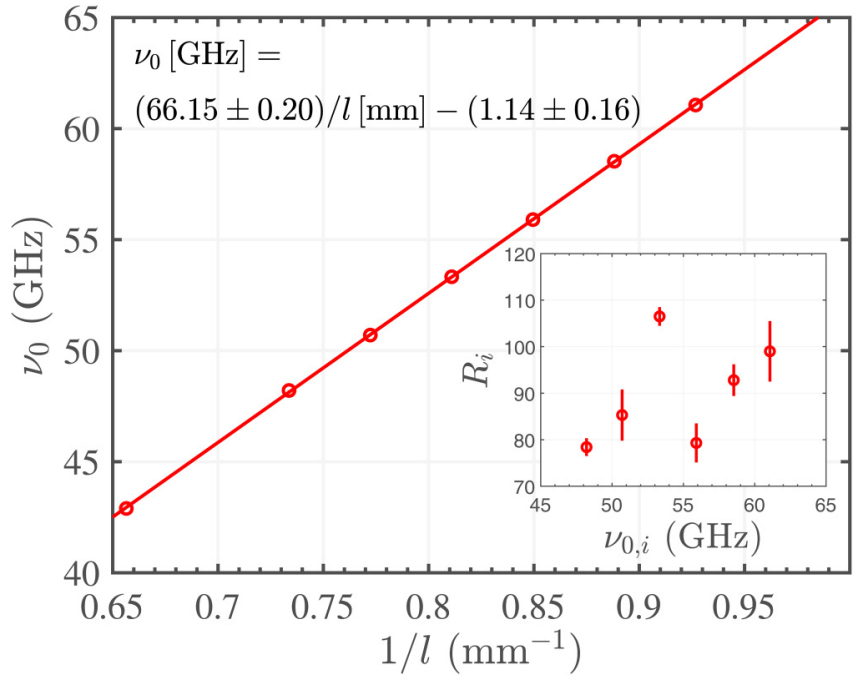

FIG. 12. Plot of measured filter center frequency as a function of inverse filter length. The inset shows the measured channel resolutions $\mathcal{R}_{i}$ with frequency.

should only affect the constant of proportionality in the relationship $v_{0} \propto l^{-1}$, rather than generate an offset. Instead, the offset suggests an issue with some detail of the underlying circuit model.

\section{SUMMARY AND CONCLUSIONS}

We have described the design, fabrication, and characterization of a superconducting filter-bank spectrometer with transition edge sensor readout. We have described the design of a waveguidemicrostrip radial probe transition with wide bandwidth. We have demonstrated the detection of millimeter-wave radiation with frequencies from 41 to $64 \mathrm{GHz}$ with characteristics that are already suitable for atmospheric temperature sounding using the $\mathrm{O}_{2}$ absorption band. The temperature of a cryogenic blackbody was modulated to determine the power detection efficiency of these prototype devices, finding an efficiency $\eta=0.91_{-0.05}^{+0.015}$ using the measured impedance of the TES to determine the detection responsivity. Filter profiles were determined on a separate device. The measured channel profiles were Lorenztian as expected. We measured extremely good predictability of relative channel placement and also in individual channel placement $\delta v / v=0.07 \%$. We found somewhat lower filter resolutions $\mathcal{R}$ than the designed values, possibly arising from our use in the filter design of dielectric constant measurements at lower frequencies compared to those explored here or, perhaps, unexpected dielectric losses. From the measured $\mathrm{RRR}$ of our $\mathrm{Nb}$ films, we estimate an effective penetration depth $\lambda_{\text {eff }}=100 \mathrm{~nm}$ so that the influence of coupling and loss into the underlying $\mathrm{SiN}_{\mathrm{x}}$ cannot be discounted. This will be an area for future investigation. In the next phase of this work, we will continue to investigate this question but also investigate alternative filter architectures-particularly coplanar structures where dielectric losses may-well be lower. Guided by the measurements reported, we will refine our designs to increase channel density for the $\mathrm{O}_{2}$-band observations and also include higher frequency channels particularly aimed at observation of the $183 \mathrm{GHz}$ water-vapor line to enable a complete on-chip atmospheric temperature and humidity sounding instrument. Finally, we emphasize that, although we have presented and discussed these measurements in the context of an enhanced atmospheric temperature sounding demonstrator, we believe we have already shown impressive spectroscopic and low-noise power detection at technically challenging millimeter-wave frequencies with applications across a broad spectrum of scientific enquiry.

\section{ACKNOWLEDGMENTS}

This work was funded by the UK Center for Earth Observation Instrumentation (CEOI) Grant No. RP61060435A07.

\section{DATA AVAILABILITY}

The data that support the findings of this study are openly available in the Cambridge Research Repository Apollo at https:/ doi.org/10.17863/CAM.52378.

\section{REFERENCES}

${ }^{1}$ B. J. Koopman, N. F. Cothard, S. K. Choi, K. T. Crowley, S. M. Duff, S. W. Henderson, S. P. Ho, J. Hubmayr, P. A. Gallardo, F. Nati, M. D. Niemack, S. M. Simon, S. T. Staggs, J. R. Stevens, E. M. Vavagiakis, and E. J. Wollack, "Advanced ACTPol low-frequency array: Readout and characterization of prototype 27 and $39 \mathrm{GHz}$ transition edge sensors," J. Low Temp. Phys. 193, 1103-1111 (2018).

${ }^{2}$ Z. Pan, P. A. R. Ade, Z. Ahmed, A. J. Anderson, J. E. Austermann, J. S. Avva, R. B. Thakur, A. N. Bender, B. A. Benson, J. E. Carlstrom, F. W. Carter, T. Cecil, C. L. Chang, J. F. Cliche, A. Cukierman, E. V. Denison, T. de Haan, J. Ding, M. A. Dobbs, D. Dutcher, W. Everett, A. Foster, R. N. Gannon, A. Gilbert, J. C. Groh, N. W. Halverson, A. H. Harke-Hosemann, N. L. Harrington, J. W. Henning, G. C. Hilton, W. L. Holzapfel, N. Huang, K. D. Irwin, O. B. Jeong, M. Jonas, T. Khaire, A. M. Kofman, M. Korman, D. Kubik, S. Kuhlmann, C. L. Kuo, A. T. Lee, A. E. Lowitz, S. S. Meyer, D. Michalik, J. Montgomery, A. Nadolski, T. Natoli, H. Nguyen, G. I. Noble, V. Novosad, S. Padin, J. Pearson, C. M. Posada, A. Rahlin, J. E. Ruhl, L. J. Saunders, J. T. Sayre, I. Shirley, E. Shirokoff, G. Smecher, J. A. Sobrin, A. A. Stark, K. T. Story, A. Suzuki, Q. Y. Tang, K. L. Thompson, C. Tucker, L. R. Vale, K. Vanderlinde, J. D. Vieira, G. Wang, N. Whitehorn, V. Yefremenko, K. W. Yoon, and M. R. Young, "Optical characterization of the SPT-3G camera," J. Low Temp. Phys. 193, 305-313 (2018).

${ }^{3}$ N. Stebor, P. Ade, Y. Akiba, C. Aleman, K. Arnold, C. Baccigalupi, B. Barch, D. Barron, S. Beckman, A. Bender, D. Boettger, J. Borrill, S. Chapman, Y. Chinone, A. Cukierman, T. de Haan, M. A. Dobbs, A. Ducout, R. Dunner, T. Elleflot, J. Errard, G. Fabbian, S. Feeney, C. Feng, T. Fujino, G. Fuller, A. J. Gilbert, N. Goeckner-Wald, J. Groh, G. Hall, N. Halverson, T. Hamada, M. Hasegawa, K. Hattori, M. Hazumi, C. Hill, W. L. Holzapfel, Y. Hori, L. Howe, Y. Inoue, F. Irie, G. Jaehnig, A. Jaffe, O. Jeong, N. Katayama, J. P. Kaufman, K. Kazemzadeh, B. G. Keating, Z. Kermish, R. Keskitalo, T. Kisner, A. Kusaka, M. Le Jeune, A. T. Lee, D. Leon, E. V. Linder, L. Lowry, F. Matsuda, T. Matsumura, N. Miller, J. Montgomery, M. Navaroli, H. Nishino, H. Paar, J. Pelotonh, D. Poletti, G. Puglisi, C. R. Raum, G. M. Rebeiz, C. L. Reichardt, P. L. Richard, C. Ross, K. M. Rotermund, Y. Segawa, B. D. Sherwin, I. Shirley, P. Siritanasak, L. Steinmetz, R. Stompor, A. Suzuki, O. Tajima, S. Takadav, S. Takatori, G. P. Teply, A. Tikhomirov, T. Tomaru, B. Westbrook, N. Whitehorn, A. Zahn, and O. Zahn, "The Simons array CMB polarization experiment," in Millimeter, Submillimeter, and Far-infrared Detectors and 
Instrumentation for Astronomy VIII, edited by W. S. Holland and J. Zmuidzinas (SPIE Proceedings, 2016), Vol. 9914.

${ }^{4}$ P. K. Grimes, K. Asada, R. Blundell, R. Burgos, H.-H. Chang, M. T. Chen, D. Goldie, C. Groppi, C. C. Han, P. T. P. Ho, Y. D. Huang, M. Inoue, D. Kubo, P. Koch, J. Leech, E. D. L. Acedo, P. Martin-Cocher, H. Nishioka, M. Nakamura, S. Matsushita, S. N. Paine, N. Patel, P. Raffin, W. Snow, T. K. Sridharan, R. Srinivasan, C. N. Thomas, E. Tong, M.-J. Wang, C. Wheeler, S. Withington, G. Yassin, and L.-Z. Zeng, "Instrumentation for single-dish observations with the Greenland Telescope," in Millimeter, Submillimeter, and Far-Infrared Detectors and Instrumentation for Astronomy VII, edited by W. S. Holland and J. Zmuidzinas (SPIE Proceedings, 2014), Vol. 9153, p. 91531V.

${ }^{5}$ A. Endo, K. Karatsu, A. P. Laguna, B. Mirzaei, R. Huiting, D. J. Thoen, V. Murugesan, S. J. C. Yates, J. Bueno, N. van Marrewijk, S. Bosma, O. Yurduseven, N. Llombart, J. Suzuki, M. Naruse, P. J. de Visser, P. P. van der Werf, T. M. Klapwijk, and J. J. A. Baselmans, "Wideband on-chip terahertz spectrometer based on a superconducting filterbank," J. Astron. Telesc. Inst. 5, 035004 (2019).

${ }^{6}$ J. Redford, J. Wheeler, K. Karkare, S. Hailey-Dunsheath, C. M. Bradford, E. Shirokoff, S. Barry, G. Che, J. Glenn, H. G. Leduc, P. Mauskopf, R. McGeehan, T. Reck, and J. Zmuidzinas, "The design and characterization of a 300 channel, optimized full-band millimeter filterbank for science with superspec," in Millimeter, Submillimeter, and Far-infrared Detectors and Instrumentation for Astronomy IX, edited by J. Zmuidzinas and J.-R. Gao (SPIE Proceedings, 2018).

${ }^{7}$ S. Zhao, D. J. Goldie, S. Withington, and C. N. Thomas, "Exploring the performance of thin-film superconducting multilayers as kinetic inductance detectors for low-frequency detection," Supercond. Sci. Technol. 31, 015007 (2018).

${ }^{8}$ P. Khosropanah, E. Taralli, L. Gottardi, C. P. de Vries, K. Nagayoshi, M. L. Ridder, H. Akamatsu, M. P. Bruijn, and J. R. Gao, "Development of TiAu TES x-ray calorimeters for the X-IFU on ATHENA space observatory," in Space Telescopes and Instrumentation 2018: Ultraviolet to Gamma Ray, edited by J. W. A. DenHerder, S. Nikzad, and K. Nakazawa (SPIE Proceedings, 2018), Vol. 10699.

${ }^{9}$ S. W. Henderson, R. Allison, J. Austermann, T. Baildon, N. Battaglia, J. A. Beall, D. Becker, F. De Bernardis, J. R. Bond, E. Calabrese, S. K. Choi, K. P. Coughlin, K. T. Crowley, R. Datta, M. J. Devlin, S. M. Duff, J. Dunkley, R. Dunner, A. van Engelen, P. A. Gallardo, E. Grace, M. Hasselfield, F. Hills, G. C. Hilton, A. D. Hincks, R. Hlozek, S. P. Ho, J. Hubmayr, K. Huffenberger, J. P. Hughes, K. D. Irwin, B. J. Koopman, A. B. Kosowsky, D. Li, J. McMahon, C. Munson, F. Nati, L. Newburgh, M. D. Niemack, P. Niraula, L. A. Page, C. G. Pappas, M. Salatino, A. Schillaci, B. L. Schmitt, N. Sehgal, B. D. Sherwin, J. L. Sievers, S. M. Simon, D. N. Spergel, S. T. Staggs, J. R. Stevens, R. Thornton, J. Van Lanen, E. M. Vavagiakis, J. T. Ward, and E. J. Wollack, "Advanced ACTPol cryogenic detector arrays and readout,” J. Low Temp. Phys. 184, 772-779 (2016).

${ }^{10}$ S. W. Henderson, Z. Ahmed, J. Austermann, D. Becker, D. A. Bennett, D. Brown, S. Chaudhuri, H.-M. S. Cho, J. M. D’Ewart, B. Dober, S. M. Duff, J. E. Dusatko, S. Fatigoni, J. C. Frisch, J. D. Gard, M. Halpern, G. C. Hilton, J. Hubmayr, K. D. Irwin, E. D. Karpel, S. S. Kernasovskiy, S. E. Kuenstner, C.-L. Kuo, D. Li, J. A. B. Mates, C. D. Reintsema, S. R. Smith, J. Ullom, L. R. Vale, D. D. Van Winkle, M. Vissers, and C. Yu, "Highly-multiplexed microwave SQUID readout using the SLAC microresonator radio frequency (SMuRF) electronics for future CMB and sub-millimeter surveys," in Millimeter, Submillimeter, and Far-Infrared Detectors and Instrumentation for Astronomy IX (SPIE Proceedings, 2018), Vol. 10708.

${ }^{11}$ K. D. Irwin, S. Chaudhuri, H. M. Cho, C. Dawson, S. Kuenstner, D. Li, C. J. Titus, and B. A. Young, "A spread-spectrum SQUID multiplexer," J. Low Temp. Phys. 193, 476-484 (2018).

${ }^{12}$ F. Aires, C. Prigent, E. Orlandi, M. Milz, P. Eriksson, S. Crewell, C.-C. Lin, and V. Kangas, "Microwave hyperspectral measurements for temperature and humidity atmospheric profiling from satellite: The clear-sky case," J. Geophys. Res. Atmos. 120, 11-334 (2015).

${ }^{13}$ P. K. Dongre, S. Havemann, P. Hargrave, A. Orlando, R. Sudiwala, S. Withington, C. Thomas, and D. Goldie, "Information content analysis for a novel TES-based hyperspectral microwave atmospheric sounding instrument," in Remote Sensing of Clouds and the Atmosphere XXIII, edited by A. Comeron, E. I. Kassianov, K. Schafer, R. H. Picard, and K. Weber (SPIE Proceedings, 2018), Vol. 10786, p. 1078608

${ }^{14}$ World Meteorological Organization, see https://www.wmo-sat.info/oscar/ instruments/view/207 for “OSCAR Instrument Guide: IASI."

${ }^{15}$ AIRS Project, see https://microwavescience.jpl.nasa.gov/files/mws/ATBDMW-21.pdf for "Algorithm Theoretical Basis Document Level 1b, Part 3: Microwave Instruments" (2000).

${ }^{16}$ G. Alberti, A. Memoli, G. Pica, M. Santovito, B. Buralli, S. Varchetta, S. d'Addio, and V. Kangas, "Two microwave imaging radiometers for metop second generation," in 2012 Tyrrhenian Workshop on Advances in Radar and Remote Sensing (IEEE, 2012), pp. 243-246.

${ }^{17}$ P. Hargrave, S. Withington, S. A. Buehler, L. Kluft, B. Flatman, and P. K. Dongre, "THz spectroscopy of the atmosphere for climatology and meteorology applications," in Next-Generation Spectroscopic Technologies X, edited by M. A. Druy, R. A. Crocombe, S. M. Barnett, and L. T. M. Profeta (SPIE Proceedings, 2017), Vol. 10210.

${ }^{18} \mathrm{G}$. Yassin and $\mathrm{S}$. Withington, "Electromagnetic models for superconducting millimetre-wave and sub-millimetre-wave microstrip transmission lines," J. Phys. D Appl. Phys. 28, 1983-1991 (1995).

${ }^{19}$ J. Kooi, G. Chattopadhyay, S. Withington, F. Rice, J. Zmuidzinas, C. Walker, and G. Yassin, "A full-height waveguide to thin-film microstrip transition with exceptional RF width and coupling efficiency," Int. J. Infrared Millimeter Waves 24, 261-284 (2003).

${ }^{20} \mathrm{~T}$. Liebig, see https://github.com/thliebig/openEMS-Project for "openEMSOpen Electromagnetic Field Solver."

${ }^{21}$ J. Zmuidzinas, "Superconducting microresonators: Physics and applications," Annu. Rev. Condens. Matter Phys. 3, 169-214 (2012).

${ }^{22}$ K. D. Irwin and G. C. Hilton, "Transition-edge sensors," in Cryogenic Particle Detection, Topics in Applied Physics, edited by C. Enss (Springer, Berlin, 2005), pp. 63-149.

${ }^{23}$ S. Zhao, D. J. Goldie, C. N. Thomas, and S. Withington, "Calculation and measurement of critical temperature in thin superconducting multilayers," Supercond. Sci. Technol. 31, 105004 (2018).

${ }^{24}$ Infinite Electronics International, Inc., see https://www.fairviewmicrowave. $\mathrm{com} /$ for details of the matched load.

${ }^{\mathbf{2 5}}$ Coax Co. Ltd., see http://www.coax.co.jp/en/product/sc/219-50-ss-ss for details of the coax cables.

${ }^{26}$ Lake Shore Cryotronics, Inc., see https://www.lakeshore.com/ for details of the $\mathrm{RuO}_{\mathrm{x}}$ thermometer.

${ }^{27}$ V. Yefremenko, P. A. R. Ade, Z. Ahmed, A. J. Anderson, J. E. Austermann, J. S. Avva, R. B. Thakur, A. N. Bender, B. A. Benson, J. E. Carlstrom, F. W. Carter, T. Cecil, C. L. Chang, J.-F. Cliche, A. Cukierman, E. V. Denison, T. de Haan, J. Ding, R. N. S. Divan, M. A. Dobbs, D. Dutcher, W. Everett, A. Foster, R. N. Gannon, A. Gilbert, J. Groh, N. W. Halverson, A. H. Harke-Hosemann, N. L. Harrington, J. W. Henning, G. C. Hilton, W. L. Holzapfel, N. Huang, K. D. Irwin, O. B. Jeong, M. Jonas, T. Khaire, A. M. Kofman, M. Korman, D. L. Kubik, S. Kuhlmann, C.-L. Kuo, A. T. Lee A. E. Lowitz, S. S. Meyer, D. Michalik, C. S. Miller, J. Montgomery, A. Nadolski, T. J. Natoli, H. Nguyen, G. Noble, V. Novosad, S. Padin, Z. Pan, J. Pearson, C. M. Posada, A. Rahlin, J. E. Ruhl, L. J. Saunders, J. T. Sayre, I. Shirley, E. D. Shirokoff, G. Smecher, J. A. Sobrin, L. Stan, A. A. Stark, K. T. Story, A. Suzuki, Q. Y. Tang, K. L. Thompson, C. E. Tucker, L. R. Vale, K. Vanderlinde, J. D. Vieira, G. Wang, N. Whitehorn, K. W. Yoon, and M. Young, "Impact of electrical contacts design and materials on the stability of Ti superconducting transition shape," J. Low Temp. Phys. 193, 732-738 (2018).

${ }^{28}$ D. J. Goldie, A. V. Velichko, D. M. Glowacka, and S. Withington, "Towards ultra-low-noise MoAu transition edge sensors," J. Low Temp. Phys. 167, 248-253 (2012). 